\title{
Search for charged Higgs bosons in decays of top quarks
}

\section{M. Abazov, ${ }^{37}$ B. Abbott,${ }^{75}$ M. Abolins,${ }^{65}$ B. S. Acharya,${ }^{30}$ M. Adams,${ }^{51}$ T. Adams, ${ }^{49}$ E. Aguilo, ${ }^{6}$ M. Ahsan,${ }^{59}$} G. D. Alexeev, ${ }^{37}$ G. Alkhazov, ${ }^{41}$ A. Alton, ${ }^{64, *}$ G. Alverson, ${ }^{63}$ G. A. Alves, ${ }^{2}$ L. S. Ancu, ${ }^{36}$ T. Andeen,${ }^{53}$ M. S. Anzelc, ${ }^{53}$ M. Aoki,${ }^{50}$ Y. Arnoud, ${ }^{14}$ M. Arov ${ }^{60}$ M. Arthaud, ${ }^{18}$ A. Askew,${ }^{49, \dagger}$ B. Àsman, ${ }^{42}$ O. Atramentov,${ }^{49, \dagger}$ C. Avila, ${ }^{8}$ J. BackusMayes, ${ }^{82}$ F. Badaud,${ }^{13}$ L. Bagby, ${ }^{50}$ B. Baldin,${ }^{50}$ D. V. Bandurin, ${ }^{59}$ S. Banerjee, ${ }^{30}$ E. Barberis,${ }^{63}$ A.-F. Barfuss, ${ }^{15}$ P. Bargassa, ${ }^{80}$ P. Baringer, ${ }^{58}$ J. Barreto, ${ }^{2}$ J. F. Bartlett, ${ }^{50}$ U. Bassler, ${ }^{18}$ D. Bauer, ${ }^{44}$ S. Beale, ${ }^{6}$ A. Bean, ${ }^{58}$ M. Begalli, ${ }^{3}$ M. Begel, ${ }^{73}$ C. Belanger-Champagne, ${ }^{42}$ L. Bellantoni, ${ }^{50}$ A. Bellavance, ${ }^{50}$ J. A. Benitez, ${ }^{65}$ S. B. Beri, ${ }^{28}$ G. Bernardi, ${ }^{17}$ R. Bernhard, ${ }^{23}$ I. Bertram, ${ }^{43}$ M. Besançon, ${ }^{18}$ R. Beuselinck, ${ }^{44}$ V. A. Bezzubov, ${ }^{40}$ P. C. Bhat, ${ }^{50}$ V. Bhatnagar,${ }^{28}$ G. Blazey, ${ }^{52}$ S. Blessing, ${ }^{49}$ K. Bloom, ${ }^{67}$ A. Boehnlein, ${ }^{50}$ D. Boline, ${ }^{62}$ T. A. Bolton, ${ }^{59}$ E. E. Boos,${ }^{39}$ G. Borissov, ${ }^{43}$ T. Bose,${ }^{62}$ A. Brandt, ${ }^{78}$ R. Brock, ${ }^{65}$ G. Brooijmans, ${ }^{70}$ A. Bross,${ }^{50}$ D. Brown, ${ }^{19}$ X. B. Bu, ${ }^{7}$ D. Buchholz, ${ }^{53}$ M. Buehler, ${ }^{81}$ V. Buescher, ${ }^{22}$ V. Bunichev ${ }^{39}$ S. Burdin, ${ }^{43,}$ T. H. Burnett, ${ }^{82}$ C. P. Buszello, ${ }^{44}$ P. Calfayan,${ }^{26}$ B. Calpas, ${ }^{15}$ S. Calvet,${ }^{16}$ J. Cammin,${ }^{71}$ M. A. Carrasco-Lizarraga, ${ }^{34}$ E. Carrera ${ }^{49}$ W. Carvalho, ${ }^{3}$ B. C. K. Casey, ${ }^{50}$ H. Castilla-Valdez ${ }^{34}$ S. Chakrabarti, ${ }^{72}$ D. Chakraborty, ${ }^{52}$ K. M. Chan, ${ }^{55}$ A. Chandra ${ }^{48}$ E. Cheu, ${ }^{46}$ D. K. Cho, ${ }^{62}$ S. Choi, ${ }^{33}$ B. Choudhary, ${ }^{29}$ T. Christoudias, ${ }^{44}$ S. Cihangir, ${ }^{50}$ D. Claes, ${ }^{67}$ J. Clutter, ${ }^{58}$ M. Cooke, ${ }^{50}$ W. E. Cooper, ${ }^{50}$ M. Corcoran, ${ }^{80}$ F. Couderc, ${ }^{18}$ M.-C. Cousinou, ${ }^{15}$ S. Crépé-Renaudin, ${ }^{14}$ D. Cutts, ${ }^{77}$ M. Ćwiok,${ }^{31}$ A. Das, ${ }^{46}$ G. Davies, ${ }^{44}$ K. De,${ }^{78}$ S. J. de Jong,${ }^{36}$ E. De La Cruz-Burelo, ${ }^{34}$ K. DeVaughan,${ }^{67}$ F. Déliot, ${ }^{18}$ M. Demarteau, ${ }^{50}$ R. Demina,${ }^{71}$ D. Denisov, ${ }^{50}$ S. P. Denisov, ${ }^{40}$ S. Desai,${ }^{50}$ H. T. Diehl,${ }^{50}$ M. Diesburg, ${ }^{50}$ A. Dominguez, ${ }^{67}$ T. Dorland, ${ }^{82}$ A. Dubey,${ }^{29}$ L. V. Dudko, ${ }^{39}$ L. Duflot,${ }^{16}$ D. Duggan, ${ }^{49}$ A. Duperrin, ${ }^{15}$ S. Dutt, ${ }^{28}$ A. Dyshkant, ${ }^{52}$ M. Eads, ${ }^{67}$ D. Edmunds, ${ }^{65}$ J. Ellison, ${ }^{48}$ V. D. Elvira,${ }^{50}$ Y. Enari, ${ }^{77}$ S. Eno, ${ }^{61}$ M. Escalier, ${ }^{15}$ H. Evans, ${ }^{54}$ A. Evdokimov, ${ }^{73}$ V. N. Evdokimov, ${ }^{40}$ G. Facini, ${ }^{63}$ A. V. Ferapontov, ${ }^{59}$ T. Ferbel,${ }^{61,71}$ F. Fiedler, ${ }^{25}$ F. Filthaut, ${ }^{36}$ W. Fisher, ${ }^{50}$ H. E. Fisk, ${ }^{50}$ M. Fortner, ${ }^{52}$ H. Fox ${ }^{43}$ S. Fu, ${ }^{50}$ S. Fuess, ${ }^{50}$ T. Gadfort, ${ }^{70}$ C. F. Galea, ${ }^{36}$ A. Garcia-Bellido, ${ }^{71}$

V. Gavrilov, ${ }^{38}$ P. Gay, ${ }^{13}$ W. Geist, ${ }^{19}$ W. Geng, ${ }^{15,65}$ C.E. Gerber, ${ }^{51}$ Y. Gershtein, ${ }^{49, \dagger}$ D. Gillberg,,${ }^{6}$ G. Ginther, ${ }^{50,71}$

B. Gómez ${ }^{8}$ A. Goussiou, ${ }^{82}$ P. D. Grannis,${ }^{72}$ S. Greder, ${ }^{19}$ H. Greenlee, ${ }^{50}$ Z. D. Greenwood, ${ }^{60}$ E. M. Gregores,${ }^{4}$ G. Grenier ${ }^{20}$

Ph. Gris, ${ }^{13}$ J.-F. Grivaz, ${ }^{16}$ A. Grohsjean, ${ }^{18}$ S. Grünendahl,${ }^{50}$ M. W. Grünewald ${ }^{31}$ F. Guo, ${ }^{72}$ J. Guo, ${ }^{72}$ G. Gutierrez,${ }^{50}$ P. Gutierrez ${ }^{75}$ A. Haas, ${ }^{70}$ P. Haefner, ${ }^{26}$ S. Hagopian, ${ }^{49}$ J. Haley, ${ }^{68}$ I. Hall, ${ }^{65}$ R. E. Hall, ${ }^{47}$ L. Han,${ }^{7}$ K. Harder,,${ }^{45}$ A. Harel, ${ }^{71}$ J. M. Hauptman, ${ }^{57}$ J. Hays,${ }^{44}$ T. Hebbeker, ${ }^{21}$ D. Hedin, ${ }^{52}$ J. G. Hegeman, ${ }^{35}$ A. P. Heinson, ${ }^{48}$ U. Heintz, ${ }^{62}$ C. Hensel, ${ }^{24}$

I. Heredia-De LaCruz, ${ }^{34}$ K. Herner, ${ }^{64}$ G. Hesketh, ${ }^{63}$ M. D. Hildreth, ${ }^{55}$ R. Hirosky, ${ }^{81}$ T. Hoang, ${ }^{49}$ J. D. Hobbs, ${ }^{72}$

B. Hoeneisen, ${ }^{12}$ M. Hohlfeld, ${ }^{22}$ S. Hossain, ${ }^{75}$ P. Houben, ${ }^{35}$ Y. Hu, ${ }^{72}$ Z. Hubacek,${ }^{10}$ N. Huske, ${ }^{17}$ V. Hynek, ${ }^{10}$ I. Iashvili, ${ }^{69}$

R. Illingworth ${ }^{50}$ A. S. Ito, ${ }^{50}$ S. Jabeen, ${ }^{62}$ M. Jaffré, ${ }^{16}$ S. Jain,${ }^{75}$ K. Jakobs,${ }^{23}$ D. Jamin, ${ }^{15}$ R. Jesik, ${ }^{44}$ K. Johns, ${ }^{46}$

C. Johnson, ${ }^{70}$ M. Johnson, ${ }^{50}$ D. Johnston, ${ }^{67}$ A. Jonckheere, ${ }^{50}$ P. Jonsson, ${ }^{44}$ A. Juste, ${ }^{50}$ E. Kajfasz, ${ }^{15}$ D. Karmanov, ${ }^{39}$ P. A. Kasper, ${ }^{50}$ I. Katsanos, ${ }^{67}$ V. Kaushik, ${ }^{78}$ R. Kehoe, ${ }^{79}$ S. Kermiche, ${ }^{15}$ N. Khalatyan, ${ }^{50}$ A. Khanov, ${ }^{76}$ A. Kharchilava ${ }^{69}$ Y. N. Kharzheev,${ }^{37}$ D. Khatidze, ${ }^{70}$ T. J. Kim, ${ }^{32}$ M. H. Kirby, ${ }^{53}$ M. Kirsch, ${ }^{21}$ B. Klima, ${ }^{50}$ J. M. Kohli, ${ }^{28}$ J.-P. Konrath, ${ }^{23}$ A. V. Kozelov, ${ }^{40}$ J. Kraus, ${ }^{65}$ T. Kuhl,${ }^{25}$ A. Kumar, ${ }^{69}$ A. Kupco, ${ }^{11}$ T. Kurča, ${ }^{20}$ V. A. Kuzmin, ${ }^{39}$ J. Kvita, ${ }^{9}$ F. Lacroix, ${ }^{13}$ D. Lam ${ }^{55}$ S. Lammers, ${ }^{54}$ G. Landsberg,${ }^{77}$ P. Lebrun, ${ }^{20}$ W. M. Lee, ${ }^{50}$ A. Leflat,${ }^{39}$ J. Lellouch, ${ }^{17} \mathrm{~J} . \mathrm{Li},{ }^{78,},{ }^{4 \neq} \mathrm{L} . \mathrm{Li},{ }^{48} \mathrm{Q} . \mathrm{Z} . \mathrm{Li},{ }^{50}$ S. M. Lietti, ${ }^{5}$ J. K. Lim,${ }^{32}$ D. Lincoln,${ }^{50}$ J. Linnemann, ${ }^{65}$ V. V. Lipaev, ${ }^{40}$ R. Lipton, ${ }^{50}$ Y. Liu, ${ }^{7}$ Z. Liu, ${ }^{6}$ A. Lobodenko, ${ }^{41}$ M. Lokajicek, ${ }^{11}$ P. Love, ${ }^{43}$ H. J. Lubatti, ${ }^{82}$ R. Luna-Garcia,${ }^{34}{ }^{\S}$ A. L. Lyon, ${ }^{50}$ A. K. A. Maciel, ${ }^{2}$ D. Mackin, ${ }^{80}$ P. Mättig, ${ }^{27}$ R. Magaña-Villalba, ${ }^{34}$ A. Magerkurth, ${ }^{64}$ P. K. Mal, ${ }^{46}$ H. B. Malbouisson, ${ }^{3}$ S. Malik, ${ }^{67}$ V. L. Malyshev,${ }^{37}$ Y. Maravin, ${ }^{59}$ B. Martin, ${ }^{14}$ R. McCarthy, ${ }^{72}$ C. L. McGivern, ${ }^{58}$ M. M. Meijer, ${ }^{36}$ A. Melnitchouk, ${ }^{66}$ L. Mendoza, ${ }^{8}$ D. Menezes, ${ }^{52}$ P. G. Mercadante, ${ }^{5}$ M. Merkin, ${ }^{39}$ K. W. Merritt, ${ }^{50}$ A. Meyer, ${ }^{21}$ J. Meyer, ${ }^{24}$ J. Mitrevski ${ }^{70}$ N. K. Mondal, ${ }^{30}$ R. W. Moore, ${ }^{6}$ T. Moulik, ${ }^{58}$ G. S. Muanza, ${ }^{15}$ M. Mulhearn, ${ }^{70}$ O. Mundal, ${ }^{22}$ L. Mundim, ${ }^{3}$ E. Nagy, ${ }^{15}$ M. Naimuddin,${ }^{50}$ M. Narain, ${ }^{77}$ H. A. Neal, ${ }^{64}$ J. P. Negret, ${ }^{8}$ P. Neustroev, ${ }^{41}$ H. Nilsen, ${ }^{23}$ H. Nogima, ${ }^{3}$ S. F. Novaes, ${ }^{5}$ T. Nunnemann, ${ }^{26}$ G. Obrant, ${ }^{41}$ C. Ochando, ${ }^{16}$ D. Onoprienko, ${ }^{59}$ J. Orduna ${ }^{34}$ N. Oshima,${ }^{50}$ N. Osman,,${ }^{44}$ J. Osta, ${ }^{55}$ R. Otec, ${ }^{10}$ G. J. Otero y Garzón, ${ }^{1}$ M. Owen, ${ }^{45}$ M. Padilla, ${ }^{48}$ P. Padley,${ }^{80}$ M. Pangilinan, ${ }^{77}$ N. Parashar, ${ }^{56}$ S.-J. Park, ${ }^{24}$ S. K. Park, ${ }^{32}$ J. Parsons, ${ }^{70}$ R. Partridge,${ }^{77}$ N. Parua,${ }^{54}$ A. Patwa, ${ }^{73}$ G. Pawloski, ${ }^{80}$ B. Penning, ${ }^{23}$ M. Perfilov, ${ }^{39}$ K. Peters,${ }^{45}$ Y. Peters,${ }^{45}$ P. Pétroff, ${ }^{16}$ R. Piegaia, ${ }^{1}$ J. Piper, ${ }^{65}$ M.-A. Pleier, ${ }^{22}$ P. L. M. Podesta-Lerma, ${ }^{34, \|}$ V. M. Podstavkov, ${ }^{50}$ Y. Pogorelov ${ }^{55}$ M.-E. Pol, ${ }^{2}$ P. Polozov, ${ }^{38}$ A. V. Popov, ${ }^{40}$ W. L. Prado da Silva, ${ }^{3}$ S. Protopopescu, ${ }^{73}$ J. Qian, ${ }^{64}$ A. Quadt, ${ }^{24}$ B. Quinn,${ }^{66}$ A. Rakitine, ${ }^{43}$ M. S. Rangel, ${ }^{16}$ K. Ranjan, ${ }^{29}$ P. N. Ratoff, ${ }^{43}$ P. Renkel,${ }^{79}$ P. Rich, ${ }^{45}$ M. Rijssenbeek, ${ }^{72}$ I. Ripp-Baudot, ${ }^{19}$ F. Rizatdinova, ${ }^{76}$ S. Robinson, ${ }^{44}$ M. Rominsky, ${ }^{75}$ C. Royon, ${ }^{18}$ P. Rubinov,${ }^{50}$ R. Ruchti, ${ }^{55}$ G. Safronov, ${ }^{38}$ G. Sajot, ${ }^{14}$ A. Sánchez-Hernández, ${ }^{34}$

M. P. Sanders, ${ }^{26}$ B. Sanghi, ${ }^{50}$ G. Savage, ${ }^{50}$ L. Sawyer, ${ }^{60}$ T. Scanlon, ${ }^{44}$ D. Schaile, ${ }^{26}$ R. D. Schamberger, ${ }^{72}$ Y. Scheglov, ${ }^{41}$ H. Schellman, ${ }^{53}$ T. Schliephake, ${ }^{27}$ S. Schlobohm, ${ }^{82}$ C. Schwanenberger, ${ }^{45}$ R. Schwienhorst, ${ }^{65}$ J. Sekaric, ${ }^{49}$ H. Severini, ${ }^{75}$ E. Shabalina, ${ }^{24}$ M. Shamim, ${ }^{59}$ V. Shary, ${ }^{18}$ A. A. Shchukin, ${ }^{40}$ R. K. Shivpuri, ${ }^{29}$ V. Siccardi, ${ }^{19}$ V. Simak, ${ }^{10}$ V. Sirotenko, ${ }^{50}$ 
P. Skubic, ${ }^{75}$ P. Slattery, ${ }^{71}$ D. Smirnov, ${ }^{55}$ G. R. Snow, ${ }^{67}$ J. Snow, ${ }^{74}$ S. Snyder, ${ }^{73}$ S. Söldner-Rembold, ${ }^{45}$ L. Sonnenschein, ${ }^{21}$ A. Sopczak, ${ }^{43}$ M. Sosebee, ${ }^{78}$ K. Soustruznik, ${ }^{9}$ B. Spurlock, ${ }^{78}$ J. Stark, ${ }^{14}$ V. Stolin, ${ }^{38}$ D. A. Stoyanova, ${ }^{40}$ J. Strandberg, ${ }^{64}$ M. A. Strang, ${ }^{69}$ E. Strauss, ${ }^{72}$ M. Strauss, ${ }^{75}$ R. Ströhmer, ${ }^{26}$ D. Strom, ${ }^{53}$ L. Stutte, ${ }^{50}$ S. Sumowidagdo, ${ }^{49}$ P. Svoisky, ${ }^{36}$ M. Takahashi, ${ }^{45}$ A. Tanasijczuk, ${ }^{1}$ W. Taylor, ${ }^{6}$ B. Tiller, ${ }^{26}$ M. Titov, ${ }^{18}$ V. V. Tokmenin, ${ }^{37}$ I. Torchiani, $^{23}$ D. Tsybychev, ${ }^{72}$ B. Tuchming, ${ }^{18}$ C. Tully, ${ }^{68}$ P. M. Tuts, ${ }^{70}$ R. Unalan, ${ }^{65}$ L. Uvarov, ${ }^{41}$ S. Uvarov, ${ }^{41}$ S. Uzunyan, ${ }^{52}$ P. J. van den Berg, ${ }^{35}$ R. Van Kooten, ${ }^{54}$ W. M. van Leeuwen, ${ }^{35}$ N. Varelas, ${ }^{51}$ E. W. Varnes, ${ }^{46}$ I. A. Vasilyev, ${ }^{40}$ P. Verdier, ${ }^{20}$ L. S. Vertogradov, ${ }^{37}$ M. Verzocchi, ${ }^{50}$ D. Vilanova, ${ }^{18}$ P. Vint,${ }^{44}$ P. Vokac, ${ }^{10}$ M. Voutilainen, ${ }^{67,}, \mathrm{I}$ R. Wagner, ${ }^{68}$ H. D. Wahl, ${ }^{49}$ M. H. L. S. Wang, ${ }^{71}$ J. Warchol, ${ }^{55}$ G. Watts, ${ }^{82}$ M. Wayne, ${ }^{55}$ G. Weber, ${ }^{25}$ M. Weber, ${ }^{50, * *}$ L. Welty-Rieger, ${ }^{54}$ A. Wenger, ${ }^{23, \dagger}$ M. Wetstein, ${ }^{61}$ A. White, ${ }^{78}$ D. Wicke, ${ }^{25}$ M. R. J. Williams, ${ }^{43}$ G. W. Wilson, ${ }^{58}$ S. J. Wimpenny, ${ }^{48}$ M. Wobisch, ${ }^{60}$ D. R. Wood, ${ }^{63}$ T. R. Wyatt, ${ }^{45}$ Y. Xie, ${ }^{77}$ C. Xu, ${ }^{64}$ S. Yacoob, ${ }^{53}$ R. Yamada, ${ }^{50}$ W.-C. Yang, ${ }^{45}$ T. Yasuda, ${ }^{50}$ Y. A. Yatsunenko, ${ }^{37}$ Z. Ye, ${ }^{50}$ H. Yin, ${ }^{7}$ K. Yip, ${ }^{73}$ H. D. Yoo, ${ }^{77}$ S. W. Youn, ${ }^{53}$ J. Yu, ${ }^{78}$ C. Zeitnitz, ${ }^{27}$ S. Zelitch, ${ }^{81}$ T. Zhao, ${ }^{82}$ B. Zhou, ${ }^{64}$ J. Zhu, ${ }^{72}$ M. Zielinski, ${ }^{71}$ D. Zieminska, ${ }^{54}$ L. Zivkovic, ${ }^{70}$ V. Zutshi, ${ }^{52}$ and E. G. Zverev ${ }^{39}$

(D0 Collaboration)

\author{
${ }^{1}$ Universidad de Buenos Aires, Buenos Aires, Argentina \\ ${ }^{2}$ LAFEX, Centro Brasileiro de Pesquisas Físicas, Rio de Janeiro, Brazil \\ ${ }^{3}$ Universidade do Estado do Rio de Janeiro, Rio de Janeiro, Brazil \\ ${ }^{4}$ Universidade Federal do ABC, Santo André, Brazil \\ ${ }^{5}$ Instituto de Física Teórica, Universidade Estadual Paulista, São Paulo, Brazil \\ ${ }^{6}$ University of Alberta, Edmonton, Alberta, Canada; Simon Fraser University, Burnaby, British Columbia, Canada; \\ York University, Toronto, Ontario, Canada; and McGill University, Montreal, Quebec, Canada \\ ${ }^{7}$ University of Science and Technology of China, Hefei, People's Republic of China \\ ${ }^{8}$ Universidad de los Andes, Bogotá, Colombia \\ ${ }^{9}$ Center for Particle Physics, Charles University, Faculty of Mathematics and Physics, Prague, Czech Republic \\ ${ }^{10}$ Czech Technical University in Prague, Prague, Czech Republic \\ ${ }^{11}$ Center for Particle Physics, Institute of Physics, Academy of Sciences of the Czech Republic, Prague, Czech Republic \\ ${ }^{12}$ Universidad San Francisco de Quito, Quito, Ecuador \\ ${ }^{13}$ LPC, Université Blaise Pascal, CNRS/IN2P3, Clermont, France \\ ${ }^{14}$ LPSC, Université Joseph Fourier Grenoble 1, CNRS/IN2P3, Institut National Polytechnique de Grenoble, Grenoble, France \\ ${ }^{15}$ CPPM, Aix-Marseille Université, CNRS/IN2P3, Marseille, France \\ ${ }^{16}$ LAL, Université Paris-Sud, IN2P3/CNRS, Orsay, France \\ ${ }^{17}$ LPNHE, IN2P3/CNRS, Universités Paris VI and VII, Paris, France \\ ${ }^{18}$ CEA, Irfu, SPP, Saclay, France \\ ${ }^{19}$ IPHC, Université de Strasbourg, CNRS/IN2P3, Strasbourg, France \\ ${ }^{20} I P N L$, Université Lyon 1, CNRS/IN2P3, Villeurbanne, France and Université de Lyon, Lyon, France \\ ${ }^{21}$ III. Physikalisches Institut A, RWTH Aachen University, Aachen, Germany \\ ${ }^{22}$ Physikalisches Institut, Universität Bonn, Bonn, Germany \\ ${ }^{23}$ Physikalisches Institut, Universität Freiburg, Freiburg, Germany \\ ${ }^{24}$ II. Physikalisches Institut, Georg-August-Universität Göttingen, Göttingen, Germany \\ ${ }^{25}$ Institut für Physik, Universität Mainz, Mainz, Germany \\ ${ }^{26}$ Ludwig-Maximilians-Universität München, München, Germany \\ ${ }^{27}$ Fachbereich Physik, University of Wuppertal, Wuppertal, Germany \\ ${ }^{28}$ Panjab University, Chandigarh, India \\ ${ }^{29}$ Delhi University, Delhi, India \\ ${ }^{30}$ Tata Institute of Fundamental Research, Mumbai, India \\ ${ }^{31}$ University College Dublin, Dublin, Ireland \\ ${ }^{32}$ Korea Detector Laboratory, Korea University, Seoul, Korea \\ ${ }^{33}$ SungKyunKwan University, Suwon, Korea \\ ${ }^{34}$ CINVESTAV, Mexico City, Mexico \\ ${ }^{35}$ FOM-Institute NIKHEF and University of Amsterdam/NIKHEF, Amsterdam, The Netherlands \\ ${ }^{36}$ Radboud University Nijmegen/NIKHEF, Nijmegen, The Netherlands \\ ${ }^{37}$ Joint Institute for Nuclear Research, Dubna, Russia \\ ${ }^{38}$ Institute for Theoretical and Experimental Physics, Moscow, Russia \\ ${ }^{39}$ Moscow State University, Moscow, Russia \\ ${ }^{40}$ Institute for High Energy Physics, Protvino, Russia \\ ${ }^{41}$ Petersburg Nuclear Physics Institute, St. Petersburg, Russia \\ ${ }^{42}$ Stockholm University, Stockholm, Sweden, and Uppsala University, Uppsala, Sweden
}


${ }^{43}$ Lancaster University, Lancaster, United Kingdom

${ }^{44}$ Imperial College, London, United Kingdom

${ }^{45}$ University of Manchester, Manchester, United Kingdom

${ }^{46}$ University of Arizona, Tucson, Arizona 85721, USA

${ }^{47}$ California State University, Fresno, California 93740, USA

${ }^{48}$ University of California, Riverside, California 92521, USA

${ }^{49}$ Florida State University, Tallahassee, Florida 32306, USA

${ }^{50}$ Fermi National Accelerator Laboratory, Batavia, Illinois 60510, USA

${ }^{51}$ University of Illinois at Chicago, Chicago, Illinois 60607, USA

${ }^{52}$ Northern Illinois University, DeKalb, Illinois 60115, USA

${ }^{53}$ Northwestern University, Evanston, Illinois 60208, USA

${ }^{54}$ Indiana University, Bloomington, Indiana 47405, USA

${ }^{55}$ University of Notre Dame, Notre Dame, Indiana 46556, USA

${ }^{56}$ Purdue University Calumet, Hammond, Indiana 46323, USA

${ }^{57}$ Iowa State University, Ames, Iowa 50011, USA

${ }^{58}$ University of Kansas, Lawrence, Kansas 66045, USA

${ }^{59}$ Kansas State University, Manhattan, Kansas 66506, USA

${ }^{60}$ Louisiana Tech University, Ruston, Louisiana 71272, USA

${ }^{61}$ University of Maryland, College Park, Maryland 20742, USA

${ }^{62}$ Boston University, Boston, Massachusetts 02215, USA

${ }^{63}$ Northeastern University, Boston, Massachusetts 02115, USA

${ }^{64}$ University of Michigan, Ann Arbor, Michigan 48109, USA

${ }^{65}$ Michigan State University, East Lansing, Michigan 48824, USA

${ }^{66}$ University of Mississippi, University, Mississippi 38677, USA

${ }^{67}$ University of Nebraska, Lincoln, Nebraska 68588, USA

${ }^{68}$ Princeton University, Princeton, New Jersey 08544, USA

${ }^{69}$ State University of New York, Buffalo, New York 14260, USA

${ }^{70}$ Columbia University, New York, New York 10027, USA

${ }^{71}$ University of Rochester, Rochester, New York 14627, USA

${ }^{72}$ State University of New York, Stony Brook, New York 11794, USA

${ }^{73}$ Brookhaven National Laboratory, Upton, New York 11973, USA

${ }^{74}$ Langston University, Langston, Oklahoma 73050, USA

${ }^{75}$ University of Oklahoma, Norman, Oklahoma 73019, USA

${ }^{76}$ Oklahoma State University, Stillwater, Oklahoma 74078, USA

${ }^{77}$ Brown University, Providence, Rhode Island 02912, USA

${ }^{78}$ University of Texas, Arlington, Texas 76019, USA

${ }^{79}$ Southern Methodist University, Dallas, Texas 75275, USA

${ }^{80}$ Rice University, Houston, Texas 77005, USA

${ }^{81}$ University of Virginia, Charlottesville, Virginia 22901, USA

${ }^{82}$ University of Washington, Seattle, Washington 98195, USA

(Received 30 June 2009; published 30 September 2009)

We present a search for charged Higgs bosons in decays of top quarks, in the mass range $80<m_{H^{ \pm}}<$ $155 \mathrm{GeV}$, assuming the subsequent decay $H^{+} \rightarrow \tau^{+} \nu_{\tau}$ (and its charge conjugate). Using $0.9 \mathrm{fb}^{-1}$ of lepton + jets data collected with the $\mathrm{D} 0$ detector at the Fermilab Tevatron $p \bar{p}$ collider, operating at a center of mass energy $\sqrt{s}=1.96 \mathrm{TeV}$, we find no evidence for a $H^{ \pm}$signal. Hence we exclude branching ratios $B\left(t \rightarrow H^{+} b\right)>0.24$ for $m_{H^{ \pm}}=80 \mathrm{GeV}$ and $B\left(t \rightarrow H^{+} b\right)>0.19$ for $m_{H^{ \pm}}=155 \mathrm{GeV}$ at the $95 \%$ C.L.

The electroweak symmetry breaking sector of the standard model (SM) contains a single SU(2) complex scalar

\footnotetext{
*Visitor from Augustana College, Sioux Falls, SD, USA.

Visitor from Rutgers University, Piscataway, NJ, USA.

${ }^{\ddagger}$ Visitor from The University of Liverpool, Liverpool, United Kingdom.

${ }^{\S}$ Visitor from Centro de Investigacion en Computacion-IPN, Mexico City, Mexico.
}

doublet field that provides gauge-invariant generation of particle masses, with the only observable particle being the electrically neutral Higgs boson $H^{0}$ [1]. Here, we search for evidence of a richer structure. The simplest extension to the SM Higgs sector involves the addition of a second

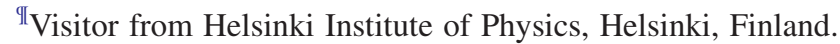

**Visitor from Universität Bern, Bern, Switzerland.

${ }^{\dagger \dagger}$ Visitor from Universität Zürich, Zürich, Switzerland.

${ }^{+}$Deceased. 
SU(2) complex scalar doublet, which introduces five spin0 particles, three that are neutral and two that are charged $\left(H^{ \pm}\right)$[2]. The fermion couplings to the Higgs doublets are not specified a priori, and the only requirement is that flavor changing neutral currents are not allowed at lowest level in perturbation theory. One possibility, the type II model, couples the up-type fermions to one Higgs doublet and the down-type fermions to the other, as required in the minimal supersymmetric extension to the SM (MSSM) [2]. In addition, the MSSM constrains the five Higgs masses through two free parameters: $\tan \beta$, the ratio of the vacuum expectation values of the two doublets, and the mass of any one of the physical Higgs bosons. We choose $m_{H^{ \pm}}$for the latter.

Since the Yukawa coupling to the $H^{ \pm}$boson increases with fermion mass for all values of $\tan \beta$, top and bottom quarks in this model are expected to have large Yukawa couplings. Therefore, if $m_{H^{ \pm}}<m_{t}-m_{b}$, the decay $t \rightarrow$ $H^{+} b$ (and its charge conjugate) is expected to have a large branching fraction for all $\tan \beta$. Further, for large values of $\tan \beta$ ( $\tan \beta \geq 10)$, the charged Higgs decays predominantly to a $\tau$ lepton and its associated neutrino with $B\left(H^{+} \rightarrow \tau^{+} \nu_{\tau}\right) \approx 1$. Hence if the $H^{ \pm}$boson exists and $B\left(t \rightarrow H^{+} b\right)$ is substantial, a search optimized for the study of SM decays of $t \bar{t}$ to lepton + jets final states should show a deficit of events relative to the SM prediction because of differences in decay branching fractions and in kinematic distributions. Any such deficit could therefore be indicative of the presence of charged Higgs bosons in decays of the top quark.

Direct searches for $H^{ \pm}$bosons have been performed at the LEP $e^{+} e^{-}$collider at CERN [3] and at the Tevatron $p \bar{p}$ collider at Fermilab [4]. With no evidence of a signal, the LEP experiments set a combined limit of $m_{H^{ \pm}}>78.6 \mathrm{GeV}$ independent of $B\left(H^{+} \rightarrow \tau^{+} \nu_{\tau}\right)$, while the Tevatron experiments have set limits in the context of a type II two Higgs doublet model that exclude regions of the $\left[\tan \beta, m_{H^{ \pm}}\right]$ parameter space [5]. Searches for indirect evidence of $H^{ \pm}$bosons through radiative decays of $B$ mesons at $B$ factories provide a combined limit of $m_{H^{ \pm}}>295 \mathrm{GeV}$ [6-8]. Although $B$ factories exclude a larger part of parameter space than our current study, it is important to search for objects such as the $H^{ \pm}$bosons through all possible channels and not defer entirely to theory.

In this article, we describe the search for charged Higgs bosons from top quark decays in $t \bar{t}$ events with one lepton (electron $e$ or muon $\mu$ ) and at least three jets. A representative Feynman diagram for such events is shown in Fig. 1, where one of the top quarks decays to a $W$ boson and a $b$ quark, as in the SM, and the other decays to a $H^{ \pm}$boson and a $b$ quark. For our signal, we consider events in which the $W$ boson decays leptonically ( $e, \mu$, or $\tau$, with the $\tau$ decaying to an $e$ or $\mu$ and two neutrinos), while the charged Higgs boson decays to a $\tau$ and a neutrino and the $\tau$ decays to a neutrino and hadrons. The final state

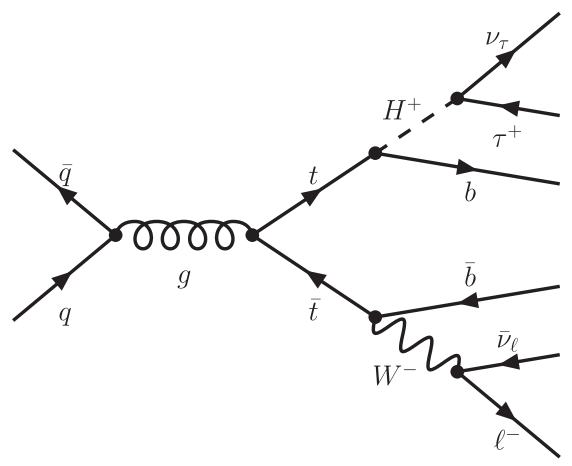

FIG. 1. Representative Feynman diagram for charged Higgs boson production in top quark decays at the Tevatron $(\ell=e$ or $\mu)$.

therefore consists of an isolated lepton ( $e$ or $\mu$ ) with large transverse momentum $\left(p_{T}\right)$, significant missing transverse energy $\left(\not \mathscr{E}_{T}\right)$ from the escaping neutrinos, and at least three jets: two from the $b$ quarks and one from the decay of the $\tau$. No attempt is made to identify $\tau$ leptons in such decays. Some of the signal can also come from events where the $\tau$ from the $H^{ \pm}$boson decays leptonically, while the $W$ boson decays into a quark-antiquark pair, thereby giving two jets. In that case, there will be four jets in the final state. Finally, if both top quarks decay into charged Higgs bosons, which then decay into $\tau$ leptons, and one $\tau$ decays leptonically while the other decays into a jet, this can also contribute to the signal. The largest backgrounds to these processes are from SM decays of $t \bar{t}$ pairs and $W+$ jets production, along with smaller contributions from the production of single top quarks, dibosons ( $W W, W Z$, and $Z Z$ ), and $Z+$ jets. An additional source of background is from multijet events, in which a jet mimics an electron or a muon from $b$ (or $c$ ) quark decay appears to be isolated.

We analyze $0.90 \pm 0.05 \mathrm{fb}^{-1}$ of data recorded with the D0 detector $[9,10]$. The trigger required a reconstructed jet and an electromagnetic energy cluster in the electron channel or a jet and a muon candidate in the muon channel. We base this analysis on a previous one that extracted the $t \bar{t}$ production cross section within the framework of the SM, i.e., assuming $B\left(t \rightarrow W^{+} b\right)=1$ [11]. The principal difference is that here we consider an additional decay mode $\left(t \rightarrow H^{+} b\right)$ and attempt to measure $B \equiv B\left(t \rightarrow H^{+} b\right)$ under the constraint $B\left(t \rightarrow W^{+} b\right)+B\left(t \rightarrow H^{+} b\right)=1$. For any measurement of $B, m_{H^{ \pm}}$is treated as a fixed parameter. Measurements are made for several values of $m_{H^{ \pm}}$.

We apply the same event selection criteria as in Ref. [11] to separate $t \bar{t}$ production from background. These are summarized in Table I. We impose an additional requirement of $\sum p_{T}(\mathrm{jet})>120 \mathrm{GeV}$ for events with only three jets and separate the events into two jet-multiplicity bins ( 3 jets and $>3$ jets) to improve signal discrimination.

To model the background distributions, $W+$ jets and $Z+$ jets events are generated using ALPGEN [13], while 
SEARCH FOR CHARGED HIGGS BOSONS IN DECAYS OF ...

TABLE I. Summary of event selections.

\begin{tabular}{lcc}
\hline \hline & $e+$ jets channel & $\mu+$ jets channel \\
\hline Lepton $(\ell)$ & $p_{T}>20 \mathrm{GeV}$ & $p_{T}>20 \mathrm{GeV}$ \\
& $|\eta|<1.1[12]$ & $|\eta|<2.0$ \\
$\mathscr{E}_{T}$ & $\mathscr{E}_{T}>20 \mathrm{GeV}$ & $\mathscr{E}_{T}>25 \mathrm{GeV}$ \\
$\Delta \phi\left(\ell, \mathscr{E}_{T}\right)[12]$ & $>0.7 \pi-0.045 \mathscr{E}_{T}$ & $>2.1 \pi-0.033 \mathscr{E}_{T}$ \\
$\left(\mathscr{E}_{T}\right.$ in $\left.\mathrm{GeV}\right)$ & & \\
Jets & $>2, p_{T}>20 \mathrm{GeV},|\eta|<2.5$ \\
& $p_{T}($ jet 1$)>40 \mathrm{GeV}$ \\
\hline \hline
\end{tabular}

SINGLETOP [14] is used for single top quark events. The events are passed through PYTHIA [15] for parton showering and hadronization. Diboson and SM $t \bar{t}$ events are generated using PYTHIA. The non-SM decay modes of $t \bar{t}$ events where one or both top quarks decay to a charged Higgs boson, are also generated using PYTHIA. The Monte Carlo (MC) events for the $H^{ \pm}$signal are produced at the following values of $m_{H^{ \pm}}: 80,100,120,140,150$, and $155 \mathrm{GeV}$. All MC generated events are processed through the D0 detector simulation based on GEANT [16], followed by application of the same reconstruction algorithms as used on D0 data. Subsequent corrections are also applied to $\mathrm{MC}$ events to account for trigger efficiencies and differences between $\mathrm{MC}$ events and data in object reconstruction efficiencies and resolutions.

To determine the number of background multijet events, we use a data sample with looser electron identification or weaker muon isolation criteria, as described in Ref. [11]. The normalization of the $W+$ jets contribution is determined differently in the current analysis, as discussed below. For the prediction of yields for the single top quark, diboson, and $Z+$ jets events, we use next-to-leading order cross sections [17]. The number of $t \bar{t}$ events is obtained by summing the different top quark decay modes according to their accepted branching fractions and respective selection efficiencies $(\epsilon)$ as follows:

$$
\begin{aligned}
N_{t \bar{t}}= & {\left[(1-B)^{2} \cdot \epsilon_{W W}+2(1-B) B \cdot \epsilon_{W H}+B^{2} \cdot \epsilon_{H H}\right] } \\
& \cdot \sigma(t \bar{t}) \cdot \int \mathcal{L} d t,
\end{aligned}
$$

where $W W$ represents SM decays of the top quark, $W H$ and $H H$ represent non-SM decays of one or both top quarks, respectively, and $\int \mathcal{L} d t$ is the integrated luminosity. We use $\sigma(t \bar{t})=7.48_{-0.72}^{+0.55} \mathrm{pb}$ for a top quark mass of $m_{t}=172.4 \pm 1.2 \mathrm{GeV}$ [18], and consider $B$ as the parameter of interest for any fixed value of $m_{H^{ \pm}}$. The selection efficiencies for the $W W$ decay modes in the different channels are $\approx 2 \%$, which includes all corrections and trigger effects. The corresponding efficiencies for the $W H(H H)$ modes vary between $1.5 \%-0.5 \%(1.2 \%-0.3 \%)$ for different values of $m_{H^{ \pm}}$.

To differentiate between $t \bar{t}$ and background, we define a multivariate discriminant
PHYSICAL REVIEW D 80, 051107(R) (2009)

$$
\mathcal{D}(\mathbf{x})=\frac{p(\mathbf{x} \mid \mathcal{S})}{p(\mathbf{x} \mid \mathcal{S})+p(\mathbf{x} \mid \mathcal{B})},
$$

where $p$ is the probability density for a set of observed variables $\mathbf{x}$, given the signal $(\mathcal{S})$ or background $(\mathcal{B})$ class of events. The signal comprises $t \bar{t}$ events which include $H^{ \pm}$ decays of the top quark and hence depends on the values of $B$ and $m_{H^{ \pm}}$. For the construction of the discriminant, we define the signal for only one value of $B$ that corresponds to the SM scenario $(B=0)$. The background is defined by all non- $t \bar{t}$ events. The variables for the different final states are listed in Table II. The normalization for the $W+$ jets template is obtained from the low- $\mathcal{D}$ region $(\mathcal{D}<0.45)$, which is background dominated, by setting the sum of all backgrounds and signal in this region to the corresponding observed number of events. We recompute the normalization for each value of $B$ and $m_{H^{ \pm}}$. The number of predicted (and observed) events for the full range of $\mathcal{D}$ appears in Table III for $B=0$. The corresponding distributions are shown for $m_{H^{ \pm}}=120 \mathrm{GeV}$ in Fig. 2 for $e+>3$ jets, for $B=0$ and $B=0.5$, in (a) and (b), respectively. We see that the data agree well with the SM predictions. Similar agreement is seen in all other channels. Hence we proceed to set upper limits on the non-SM branching fraction $B(t \rightarrow$ $\left.H^{+} b\right)$.

We use a modified frequentist approach [20] to set limits at the $95 \%$ C.L. in the high- $\mathcal{D}$ region since it is $t \bar{t}$ dominated. Sources of uncertainty on the predicted yields are included with correlations across samples and channels. Their estimated values are provided in Table IV. Note that all these uncertainties are applied to the $W+$ jets normalization assuming full anticorrelation because of the manner in which the $W+$ jets normalization is derived as explained above. The dominant sources of uncertainties are from the integrated luminosity, the jet energy calibration, and the $t \bar{t}$ cross section. The uncertainties from the normalization of multijet, single top quark, diboson, and $Z+$ jets events have a less pronounced effect on the $B$ limits because of the smaller contribution of these samples in the high discriminant region. We consider the distribution in

TABLE II. Variables used to define the discriminant $\mathcal{D} . \Delta R=$ $\sqrt{(\Delta \phi)^{2}+(\Delta \eta)^{2}}$ and $i$ indexes the list of $N_{j}$ jets ordered in decreasing $p_{T}$.

\begin{tabular}{lc}
\hline \hline Variable & Channel \\
\hline$\sum_{i \overline{N_{j}}{ }^{3}}^{N_{j}} p_{T}(i)$ & all \\
$\sum_{i \overline{N_{j}}} p_{T}(i) / \sum_{i=1}^{N_{j}} p_{z}(i)$ & $e+3$ jets, $e+>3$ jets \\
$\sum_{i=1} p_{T}(i)+p_{T}(e)+\mathbb{E}_{T}$ & $e+3$ jets, $e+>3$ jets \\
$\Delta R(\ell$, jet1 $)$ & all \\
$\Delta R($ jet1, jet2) & $e+>3$ jets, $\mu+>3$ jets \\
$\Delta \phi\left(\ell, \mathbb{E}_{T}\right)$ & $\mu+3$ jets, $\mu+>3$ jets \\
$\Delta \phi\left(\right.$ jet1, $\left.\mathbb{E}_{T}\right)$ & $e+3$ jets, $\mu+3$ jets \\
Sphericity $\mathcal{S}[19]$ & all but $\mu+3$ jets \\
Aplanarity $\mathcal{A}[19]$ & all but $\mu+3$ jets \\
\hline \hline
\end{tabular}


TABLE III. Event yields after all selections for channels separated by lepton flavor and jet multiplicity. We assume $B\left(t \rightarrow H^{+} b\right)=0$ so that $t \bar{t}$ includes only SM decays of the top quarks. The "other MC" comprises single top quark, diboson, and $Z+$ jets events. (The uncertainty on the total SM prediction includes correlations across samples.)

\begin{tabular}{lcccc}
\hline \hline Source & $e+3$ jets & $\mu+3$ jets & $e+>3$ jets & $\mu+>3$ jets \\
\hline Signal $(t \bar{t})$ & $148.8 \pm 20.0$ & $108.2 \pm 14.7$ & $130.4 \pm 19.4$ & $105.6 \pm 15.4$ \\
$W+$ jets & $535.4 \pm 47.9$ & $572.4 \pm 34.7$ & $79.2 \pm 17.3$ & $152.0 \pm 16.5$ \\
Other MC & $102.5 \pm 14.6$ & $106.7 \pm 15.3$ & $33.1 \pm 4.8$ & $35.0 \pm 5.3$ \\
Multijets & $194.2 \pm 30.5$ & $33.5 \pm 13.9$ & $60.2 \pm 10.1$ & $10.4 \pm 5.7$ \\
Total SM prediction & $980.9 \pm 25.8$ & $820.8 \pm 27.6$ & $302.9 \pm 13.1$ & $303.0 \pm 15.6$ \\
Observed & 948 & 812 & 320 & 306 \\
\hline \hline
\end{tabular}

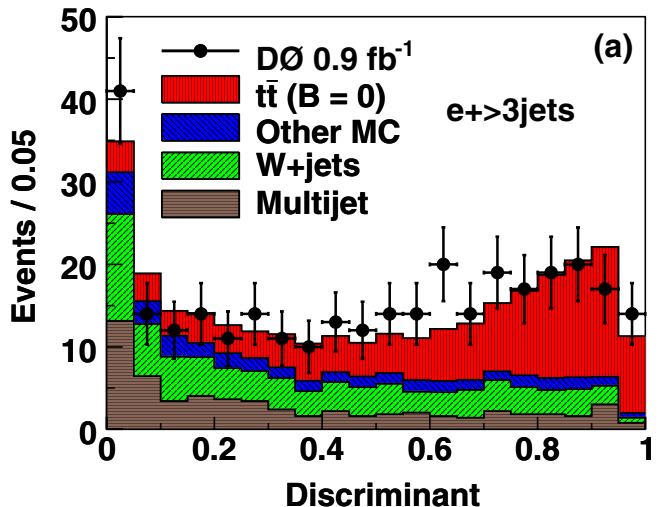

FIG. 2 (color online). Distributions in the discriminant $\mathcal{D}$ for $m_{H^{ \pm}}=$

$\mathcal{D}$ above 0.55 in the $>3$ jets channels and 0.6 in the 3 jets channels, a choice determined by maximizing the sensitivity of the analysis in MC simulation. The sensitivity is defined as the median of limits obtained from an ensemble of background plus SM $t \bar{t}(B=0)$ pseudoexperiments in each channel. We call these the expected limits and show them by the dashed curve in Fig. 3 along with their \pm 1 standard-deviation (SD) intervals by the crosshatched region. The observed limits, using D0 data, are shown by the solid curve in Fig. 3.

TABLE IV. Uncertainties (equivalent to $\pm 1 \mathrm{SD}$ ) from different components affecting the predicted yields. "Other MC" comprises single top quark, diboson, and $Z+$ jets events.

\begin{tabular}{lc}
\hline \hline Component & Uncertainty [\%] \\
\hline Integrated luminosity & 6.1 \\
Primary vertex modeling & 2.2 \\
Trigger efficiency & $0.5-2.8$ \\
Lepton identification & $2.2-2.6$ \\
Jet energy calibration & 5.0 \\
Jet identification & $2.0-2.4$ \\
Jet energy resolution & $0.1-1.8$ \\
Multijets normalization & $15.7-54.8$ \\
Other MC normalization & $11.0-12.0$ \\
$\sigma(t \bar{t})$ & $7.4-9.6$ \\
$m_{t}$ & 2.1 \\
MC statistics & $0.9-25.0$ \\
\hline \hline
\end{tabular}

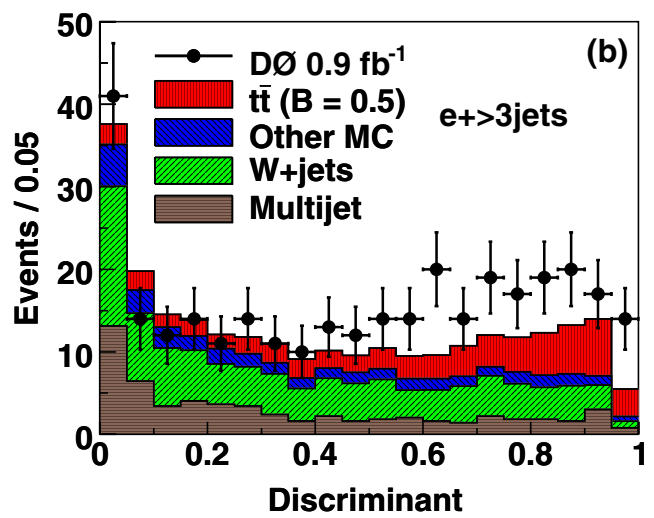

$=120 \mathrm{GeV}$ in $e+>3$ jets, for (a) $B=0$ (SM), and (b) $B=0.5$.

The upper limit on $B\left(t \rightarrow H^{+} b\right)$ can be used to exclude regions of the $\left[\tan \beta, m_{H^{ \pm}}\right]$parameter space in the context of the MSSM. Since the MSSM has several free parameters, we select them according to the $m_{h}^{\max }$ scenario described in Ref. [21]. This provides the maximum range in the mass of the lightest neutral Higgs boson as a function of $\tan \beta$. The exclusion bounds are calculated using FEYNHIGGS [22], which includes the two-loop QCD and MSSM corrections. Figure 4 shows the expected and observed excluded regions, and the theoretically inaccessible region defined as the boundary where certain Higgs parameters acquire unphysical values.

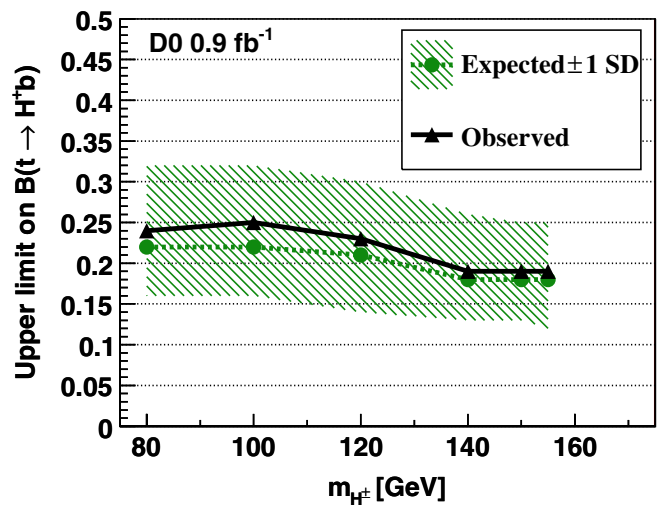

FIG. 3 (color online). The $95 \%$ C.L. limits on $B\left(t \rightarrow H^{+} b\right)$ for different values of $m_{H^{ \pm}}$. 


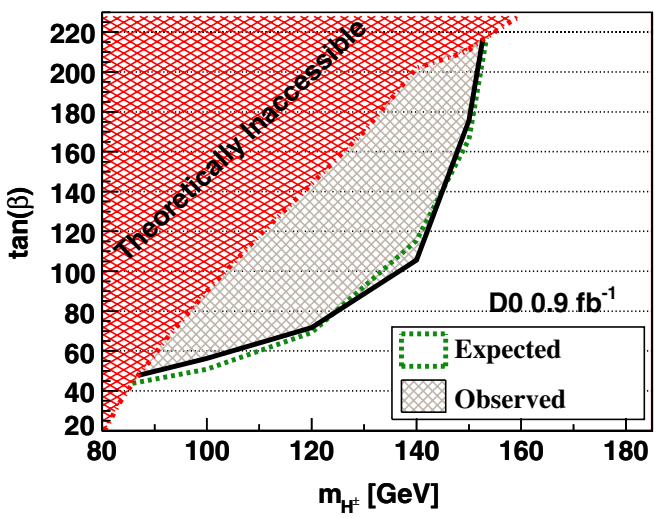

FIG. 4 (color online). The MSSM exclusion regions for the $m_{h}^{\max }$ scenario.

In summary, we have analyzed $0.90 \pm 0.05 \mathrm{fb}^{-1}$ of lepton + jets data at D0, and found no evidence for top quark decays to charged Higgs bosons. Hence we set upper limits at the $95 \%$ C.L. on $B\left(t \rightarrow H^{+} b\right)$ ranging from 0.24 for $m_{H^{ \pm}}=80 \mathrm{GeV}$ to 0.19 for $m_{H^{ \pm}}=155 \mathrm{GeV}$.

We thank the staffs at Fermilab and collaborating institutions, and acknowledge support from the DOE and NSF (USA); CEA and CNRS/IN2P3 (France); FASI, Rosatom, and RFBR (Russia); CNPq, FAPERJ, FAPESP, and FUNDUNESP (Brazil); DAE and DST (India); Colciencias (Colombia); CONACyT (Mexico); KRF and KOSEF (Korea); CONICET and UBACyT (Argentina); FOM (The Netherlands); STFC and the Royal Society (United Kingdom); MSMT and GACR (Czech Republic); CRC Program, CFI, NSERC, and WestGrid Project (Canada); BMBF and DFG (Germany); SFI (Ireland); The Swedish Research Council (Sweden); CAS and CNSF (China); and the Alexander von Humboldt Foundation (Germany).
[1] P. W. Higgs, Phys. Lett. 12, 132 (1964); Phys. Rev. Lett. 13, 508 (1964); Phys. Rev. 145, 1156 (1966); F. Englert and R. Brout, Phys. Rev. Lett. 13, 321 (1964); G.S. Guralnik, C. R. Hagen, and T. W. B. Kibble, Phys. Rev. Lett. 13, 585 (1964).

[2] J. F. Gunion, H. E. Haber, G. Kane, and S. Dawson, The Higgs Hunter's Guide (Addison-Wesley, Redwood City, CA, 1990).

[3] G. Abbiendi et al. (OPAL Collaboration), Eur. Phys. J. C 7, 407 (1999); R. Barate et al. (ALEPH Collaboration), Phys. Lett. B 543, 1 (2002); J. Abdallah et al. (DELPHI Collaboration), Phys. Lett. B 525, 17 (2002); P. Achard et al. (L3 Collaboration), Phys. Lett. B 575, 208 (2003).

[4] B. Abbott et al. (D0 Collaboration), Phys. Rev. Lett. 82, 4975 (1999); V. M. Abazov et al. (D0 Collaboration), Phys. Rev. Lett. 88, 151803 (2002); A. Abulencia et al. (CDF Collaboration), Phys. Rev. Lett. 96, 042003 (2006); V. M. Abazov et al. (D0 Collaboration), Phys. Rev. Lett. 102, 191802 (2009); V.M. Abazov et al. (D0 Collaboration), arXiv:0903.5525.

[5] W. M. Yao et al., J. Phys. G 33, 1 (2006).

[6] P. Koppenburg et al. (Belle Collaboration), Phys. Rev. Lett. 93, 061803 (2004).

[7] B. Aubert et al. (BABAR Collaboration), Phys. Rev. D 72, 052004 (2005); Phys. Rev. Lett. 97, 171803 (2006).

[8] M. Misiak et al., Phys. Rev. Lett. 98, 022002 (2007).

[9] T. Andeen et al., Report No. FERMILAB-TM-2365, 2007.

[10] V. M. Abazov et al. (D0 Collaboration), Nucl. Instrum. Methods Phys. Res., Sect. A 565, 463 (2006).

[11] V. M. Abazov et al. (D0 Collaboration), Phys. Rev. Lett. 100, 192004 (2008).

[12] The D0 coordinate system has the positive $z$ axis along the proton beam line, and $z=0$ at the center of the detector. The polar and azimuthal angles are denoted as $\theta$ and $\phi$, respectively. The pseudorapidity is defined as $\eta=$ $-\ln \left(\tan \frac{\theta}{2}\right)$.

[13] M. L. Mangano et al., J. High Energy Phys. 07 (2003) 001; S. Höche et al., arXiv:hep-ph/0602031.

[14] E. E. Boos et al., Phys. At. Nucl. 69, 1317 (2006).

[15] T. Sjöstrand et al., arXiv:hep-ph/0308153.

[16] R. Brun and F. Carminati, CERN Program Library Long Writeup, Report No. W5013, 1993.

[17] E. E. Boos et al., Phys. At. Nucl. 69, 1317 (2006); Z. Sullivan, Phys. Rev. D 70, 114012 (2004); J. M. Campbell and R. K. Ellis, Phys. Rev. D 60, 113006 (1999).

[18] S. Moch and P. Uwer, Phys. Rev. D 78, 034003 (2008); (private communications).

[19] A momentum tensor is defined as $\mathcal{M}_{i j}=$ $\Sigma_{o} p_{i}^{o} p_{j}^{o} / \Sigma_{o}\left|\vec{p}^{o}\right|^{2}$, where $\vec{p}^{o}$ is the momentum of a reconstructed object $o$, and $i$ and $j$ are Cartesian coordinates. The sum over $o$ includes up to four jets of leading $p_{T}$ and the lepton. The sphericity in an event is defined as $\mathcal{S}=$ $\frac{3}{2}\left(\lambda_{2}+\lambda_{3}\right)$, where $\lambda_{2}$ and $\lambda_{3}$ are the smallest two eigenvalues of the normalized momentum tensor. The aplanarity is defined as $\mathcal{A}=\frac{3}{2} \lambda_{3}$.

[20] T. Junk, Nucl. Instrum. Methods Phys. Res., Sect. A 434, 435 (1999).

[21] M. Carena et al., Eur. Phys. J. C 26, 601 (2003).

[22] S. Heinemeyer, W. Hollik, and G. Weiglein, Eur. Phys. J. C 9, 343 (1999); Comput. Phys. Commun. 124, 76 (2000); G. Degrassi et al., Eur. Phys. J. C 28, 133 (2003); M. Frank et al., J. High Energy Phys. 02 (2007) 047, version 2.6.5 of the code was used. 